\title{
La afición por el cine ante la cultura digital: hacia una caracterización de la cinefilia 2.0
}

\author{
Zinemarekiko zaletasuna eta kultura digitala: \\ 2.0 zinemazaletasunaren ezaugarriak
}

The fondness for cinema in digital culture: characterizing cinephilia 2.0

\author{
José Alberto Abril Valdez ${ }^{\star}$ \\ Universidad de Sonora (México)
}

RESUMEN: Durante décadas la cinefilia tuvo como paradigma la cultura cinematográfica francesa; se originó a mediados del siglo $\mathrm{xx}$, se vinculaba a un discurso erudito sobre el cine y a unos espacios específicos para su cultivación. Pero la revolución digital instauró nuevas prácticas de consumo cinematográfico e Internet catapultó nuevas cinefilias. En este artículo se analiza el papel de la cultura digital en estas nuevas cinefilias y se describe cómo los jóvenes aficionados al cine encuentran hoy los nuevos espacios virtuales para forjarla y pronunciarla.

PALABRAS CLAVE: Cinefilia; cine; estudios de audiencia; cultura fan; comunicación 2.0.

ABSTRACT: For several decades, cinephilia took its paradigm from the French film culture, which has it origins in the mid-twentieth century, and it was linked to a scholarly discourse on cinema and to very specific spaces for its cultivation. However, the digital revolution brought new consumer practices and the Internet catapulted a new crowd of cinephiles. This paper analyzes the role of digital culture in those new cinephiles and describes how young cinema fans find and use the new virtual spaces to pronounce it today.

KEYWORD: Cinephilia; cinema; audience studies; fan culture; communication 2.0.

\footnotetext{
* Correspondencia a / Corresponding author: José Alberto Abril Valdez. Universidad de Sonora (Unison). Dep. of Psychology \& Communication Studies. Blvd. Luis Encinas y Rosales, s/n, Col. Centro, Hermosillo (Sonora-México. CP 83000) - seis14@yahoo.com - https://orcid. org/0000-0003-1880-5424

Cómo citar / How to cite: Abril Valdez, José Alberto (2019). «La afición por el cine ante la cultura digital: hacia una caracterización de la cinefilia 2.0», Zer, 25(47), 105-117. (https://doi.org/10.1387/zer.20955).

Recibido: 27 junio, 2019; aceptado: 6 septiembre, 2019.

ISSN 1137-1102 - eISSN 1989-631X / (c) 2019 UPV/EHU

(c) (7) Esta obra está bajo una licencia
} 


\section{Introducción: La cultura cinematográfica y sus derivas en el tiempo}

La preocupación por la muerte del cine fue el centro de los debates estéticos a finales del siglo Xx. ¿Por qué llamar, de entrada, la atención en ello? Una mirada retrospectiva lo justifica: ante los albores del nuevo siglo resultaba enigmático —algo incongruente, habría que matizar- que muy pocos se preguntaran sobre su futuro — más allá de anatemizar su presente — más aún si se consideran las evidentes transformaciones tecnológicas que se estaban suscitando. Si el cine, desde su nacimiento, nunca se desvinculó de la tecnología y por lo mismo, durante todo el siglo pasado, no cesó de evolucionar, por qué habría de hacerlo con la llegada de la revolución digital. Hoy, los sobrevivientes analógicos seguimos constatando que el cine sigue más vivo que como en sus inicios, y que aquellas viejas dudas apocalípticas han sido desplazadas por otras más relacionadas con su estado vital: ¿En qué se ha convertido el cine?¿Hacia dónde y de qué formas ha evolucionado?

Pero aquí nos referimos al cine -y sus cambios- no solo como un dispositivo tecnológico, que es su signo más evidente, sino también como un dispositivo estético y socio-cultural. Y lo hacemos en el sentido de que a medida de que la tecnología ha ido trasformando las formas de registrar la realidad a través de la imagen, esa misma evolución tecnológica, cinematográficamente hablando, ha ido transformando, por un lado, las maneras de manipular la imagen para significar esa realidad $\mathrm{y}$, por otro, las formas y los procesos de aproximarse a ellas, de hacer cosas con ellas en tanto espectadores.

Es esta última dimensión socio-cultural — del cine y sus transformaciones- la que se ubica en el centro de este artículo. Lo que sigue, por lo tanto, no dirige su atención en el cine como dispositivo tecnológico y estético especialmente pero sí como un dispositivo de enorme implicación social. Particularmente hablamos de espectadores, pero no tanto de aquellos que se definen por una delimitación espacial muy específica como es la sala de exhibición que, sin haber sido desplazada del todo, ocupa un lugar secundario para las nuevas generaciones de aficionados cinematográficos: para estas nuevas generaciones tanto la experiencia espectatorial como la sociabilidad que de ella se desprende carecen de un arraigo espacial/territorial; son procesos que llevan hoy la impronta de la cultura digital y que se experimentan trascendiendo los entornos locales (Octobre, 2019).

Partimos de la idea de que hoy los espectadores reconvertidos en usuarios reconfiguran nuevas maneras de dialogar entre sí, interactuar y establecer vínculos mediante los usos de lo audiovisual, del cine, de las películas, en el ámbito online; a los tradicionales hábitos de consumo cinematográfico (acudir a una sala de cine o cineclub, estar frente a una televisión en una sala doméstica o en una habitación) se le 
han sumado nuevas maneras de estar frente a la imagen, de relacionarse con y apropiarse de ella.

Una anécdota resultado de nuestro proceder metodológico resultaría ilustrativa como parte de este planteamiento. «Mi gusto por el cine se forjó descargando torrents» nos comentó en una entrevista una muy joven realizadora argentina (de la que omitimos su nombre por petición de la propia informante), con una ironía que intentaba innecesariamente disfrazar mucho de su honestidad, una honestidad que no debería tener estigma alguno.

La confesión resulta por demás significativa si vamos más allá de su enunciación: no sólo es la explicación directa de un muy generalizado modus operandi, aún incluso en nuestra era del streaming, sino también la expresión clara y pura del cambio de paradigma relacionado con nuestro tema de interés, la cinefilia, entendida ésta como una modalidad de recepción cinematográfica (Sedeño, 2013). Para comprender ese cambio de paradigma (el de cómo se forja el gusto por el cine hoy) se ha introducido el concepto de cinefilia 2.0 o cinefilia posmoderna (Jullier y Leveratto, 2012). Contribuir a la comprensión de esos procesos es parte de nuestros propósitos.

Al respecto Stam (2001) nos recuerda que todo análisis contemporáneo de los procesos de comunicación audiovisual, de consumo y espectatorialidad, debe tomar en consideración no solo los nuevos espacios de visionado, sino también el hecho de que las nuevas tecnologías audiovisuales han generado un nuevo tipo de audiencia. Por ello, se propone en adelante analizar la cinefilia 2.0, en tanto práctica cultural, a partir de las intersecciones entre dos dimensiones centrales: la cultura cinematográfica y la cultura digital, además de describir a esta nueva cinefilia bajo el paradigma de la cultura fan.

\section{Sobre el concepto de cinefilia}

En los últimos años, gracias principalmente al marketing, palabras como cinefilia o cinéfilo/a empezaron a volverse de uso frecuente. Ello provocó el sentido erróneo atribuido a esos términos vinculándolos a una reiterada visita a las salas de cine o acceso constante a las plataformas audiovisuales de servicio streaming. Es importante matizar al respecto: efectivamente, todo mundo es potencialmente espectador, en tanto que se asiste con una frecuencia relativa al cine o, con la misma frecuencia relativa, vemos películas online, pero el hecho de que se desarrollen esos hábitos de consumo no significa que todos los espectadores deban definirse bajo el concepto de cinefilia o se identifiquen como cinéfilos.

El cineasta Elia Kazan (1909-2003) solía decir que el cine es el diálogo del mundo. Intentemos explicar, bajo la lógica de la metáfora de Kazan, qué sentido se 
le atribuye a la cinefilia: si el cine es el diálogo del mundo, hay solo una parte de ese mundo que pone especial interés en aumentar la intensidad - emocional, intelectual, cognitiva- de ese diálogo. Conversar con el cine y mantener conversaciones a partir de él es sustancial a la cinefilia, de aquí que se le suela considerar, como se señalaba anteriormente, una manera distinta y algo especial de recepción cinematográfica. Dicho de esta forma la palabra designaría:

[...] a la cultura cinematográfica, en el doble sentido de un saber adquirido por la experiencia de las películas y de una acción de cultivar el placer cinematográfico. Ella abarca al mismo tiempo la memoria y la capacidad de juzgar adquirida al contacto de una técnica artística frecuentada durante nuestro esparcimiento [...]. (Jullier y Leveratto, 2012, p. 11)

Se trata por un lado de un conocimiento adquirido por contacto con las películas y, por otro, de todo lo que hacemos para cultivar - y socializar- ese conocimiento y ese gusto. Implica una posición ritualizada frente al cine, una serie de narrativas que se van construyendo en torno a él que permiten prolongar el placer y los discursos que se configuran en los procesos de socialización de ese placer (Jullier y Leveratto, 2012).

Desde la perspectiva de Pujol (2011) es menos complicado explicar la cinefilia si atendemos los ritos que la constituyen. De esta manera la palabra define tanto a un grupo de personas que mantienen un fuerte vínculo con el cine como a una serie de prácticas que fortalecen esa vinculación. Específicamente, según la autora, se trata de un conjunto de espectadores caracterizados y diferenciados de otros por una relación estrecha, exacerbada, intelectual a la vez que sentimental con el cine, relación que se reafirma, se significa y traduce mediante el desarrollo de una serie de hábitos un tanto colaterales a los procesos de expectación.

En ese sentido el cinéfilo es un espectador que procura enriquecer sus procesos como espectador con la documentación, es decir leer e informarse sobre historia del cine, sobre determinadas películas, directores y géneros; intenta ampliar el goce a través del coleccionismo, que es una forma de apropiación de todo aquel producto cinematográfico que sea de su agrado, mediante el videocasete o DVD antes de la llegada de Internet, o bien de la producción que de él se desprende, como la publicidad impresa, lo que se escribe en la prensa, etc.

Pero la práctica que sin duda legitima la cinefilia del espectador, lo que le da visibildad es la socialización de ese gusto que implica el intercambio de juicios cinematográficos, estéticos, y opiniones a través de la asistencia a tertulias o foros de debates, funciones de cineclubes, conversaciones improvisadas o formalizadas mediante textos críticos que se integran a procesos de interacción. 
Vista de esta forma la cinefilia es el factor identitario de una comunidad interpretativa integrada por un determinado tipo de audiencia que adopta una manera particular de aproximarse a las películas, mantiene conversaciones reflexivas sobre ellas y busca las formas de difundir ese discurso (Pujol, 2011; Jullier y Leveratto, 2012; Sedeño, 2013); ello implica también determinados procesos de aprendizaje, la mayoría de las veces de carácter informal, sobre historia, técnica, narrativa y estética cinematográficas. Se trata, en ese sentido, de «la conjunción de un saber compartido y de una actividad de intercambio, generada por la afición de una técnica artística como la cinematográfica». (Jullier y Leveratto, 2012, p.19).

Durante varias décadas hubo una cinefilia marcada por la cultura cinematográfica francesa. Tuvo su origen a finales de los cincuenta y los primeros sesenta - particularmente sujeta a las preocupaciones estéticas y políticas del movimiento llamado Nueva Ola Francesa-, solía definírsele como una mirada fascinada hacia el cine, que daba centralidad al placer en lo espectatorial reconociendo a su vez los procesos cognitivos que lo incluye, se le asociaba, además, a unos espacios muy específicos para su cultivación (las cinetecas, los cineclubes, las revistas de crítica cinematográfica, la realización cinematográfica propiamente).

En ese primer momento a la cinefilia, que tuvo su espacio de enunciación en la revista Cahiers du Cinéma y de encuentro en las sesiones de la Cinemateca francesa, le era propia la legitimación estética de un cine considerado menor (el cine de géneros hollywoodense) en oposición a un cine academicista; como tal esta cinefilia representada por la Nueva Ola Francesa supuso un elemento que dinamitaba la distinción cultural entre las artes creando un espacio entre el gran arte y las artes populares y difuminando los límites entre géneros del entretenimiento (Sedeño. 2013).

Una cinefilia fuertemente politizada que marcaba su distancia con la muy menospreciada cultura de masas (las industrias consolidadas como tal en general; la industria de Hollywood en particular) será la propia de un segundo momento. Es esta cinefilia como erudición y distinción la que será producto de su institucionalización, de su integración a la cultura universitaria, la que toma como base los estudios académicos para legitimarse y que se posicionará durante la segunda mitad del siglo Xx. Es esa cinefilia la que se mantuvo como paradigma hasta los albores del presente siglo (Pujol, 2011; Jullier y Leveratto, 2012; Sedeño, 2013).

De acuerdo con Pujol (2011), bajo este último paradigma, la cinefilia mantendría unos rasgos característicos importantes de tomar en cuenta: era clasemediera, heterosexualmente masculina y, principalmente, joven (jóvenes con acceso a la educación superior). En ese orden de ideas, el espectador se asume cinéfilo — se vuelve consciente de ello- en la transición de dos etapas escolares, de la educación media superior a la educación superior, por dos factores: 1). es la etapa en la que el joven 
empieza a ser adulto, y 2). el ambiente académico que fomenta y propicia actividades relacionadas con ello (actividades de cineclubes, cine debates, los conocimientos adquiridos que van ampliando su visión sobre las cosas).

Por lo anterior, nos plantea Pujol, la cinefilia — durante la segunda mitad del siglo $\mathrm{xx}$ - fue sinónimo de status y se consideraba una filia algo elitista, una práctica exclusiva de jóvenes hombres de clase media ilustrada en el que el gusto femenino prácticamente no se tomaba en cuenta. Pero, concluye Pujol, la llegada de internet transformó radicalmente esa idea de cinefilia y esa concepción. La sociedad de la información, la convergencia de medios, la relativa democratización del consumo cultural vía el ciberespacio popularizaron la cinefilia, la pluralizaron, la diversificaron. Y la aumentaron.

\section{La cultura cinematográfica en diálogo con la cultura digital}

De acuerdo con Carlos Scolari (2008):

Cada época genera sus tecnologías, que a su vez contribuyen a definir ese momento histórico [...] Las tecnologías no sólo transforman al mundo sino que también influyen en la percepción que los sujetos tienen de ese mundo. (p. 273)

En ese sentido cabe preguntarse: bajo este contexto de la convergencia y sociedad digital ¿En qué sentido se ha transformado, particularmente, la cultura cinematográfica y los procesos y prácticas que la constituyen? Ello conduce a puntualizar también — quizá en primer término- las transformaciones que ha sufrido una figura clave en dichos procesos, el espectador definido, en nuestro caso particular, bajo el signo de la cinefilia. Estas transformaciones van a la par con el desarrollo mismo de la tecnología de registro y reproducción de las imágenes cinematográficas que le provee siempre de renovadas condiciones para aproximarse a ellas. A consideración de Azatto Sordo (2011):

Conforme han aparecido diversas tecnologías visuales, la pantalla ha evolucionado en el tiempo y con ello también el observador que la visualiza adaptándose a cada nuevo régimen visual. (p. 84)

Por ello se considera que hoy es fundamental y necesario explicar la cinefilia en diálogo con la cultura digital porque al entrar en contacto con ésta la cinefilia se transformó y puso en tensión el ya viejo paradigma (Pujol, 2011). De acuerdo con Ardèvol et al (2010), la cultura digital nos remite un conjunto de procesos emergentes que se generan a partir de interacciones complejas entre las tecnologías digitales y las infraestructuras en red; es decir, un conjunto amplio y variado de prácticas, dis- 
positivos materiales y narrativas relacionadas con la producción cultural contemporánea que tiene su origen en los usos de las tecnologías digitales de la comunicación y la información.

La cultura cinematográfica de cara a la cultura digital supone cambios sustanciales. Cuando hablamos de una nueva cultura cinematográfica y, por ende, audiovisual, nos referimos a una serie de distanciamientos con una cultura audiovisual precedente. Una cultura audiovisual previa definida por lo analógico, transformada ahora a raíz de lo digital, renovada constantemente a medida que se amplían y diversifican los hábitos de creación y producción, de puesta en circulación, como de recepción y consumo.

Lo digital le ha dado un carácter ubicuo a la imagen, y en general a los bienes culturales (Brea, 2007; Bourriaud, 2009), y esa ubicuidad ha posibilitado el surgimiento de diversas y novedosas formas de producir, crear, difundir o divulgar información, y por lo tanto nuevos hábitos de consumir y socializar a través de lo mediático (Gómez, 2015b). Esas rupturas con una serie de tradiciones ligadas a lo cinematográfico, a lo audiovisual han encontrado su caldo de cultivo en el ciberespacio con el auxilio de una gran variedad de dispositivos tecnológicos.

Una de esas tantas rupturas se relaciona con la forma de acercarse, como espectador, al cine - uno de los varios dialectos constituyentes de esta nueva cultura audiovisual, de acuerdo con Gubern (2000)—. A lo largo de su historia el cine ha estado vinculado a lo colectivo, a lo grupal en varios sentidos. Desde su surgimiento, a finales del siglo diecinueve, hasta la actualidad el espectador ha organizado sus formas de acceso a las representaciones audiovisuales mediante rituales colectivos espacialmente delimitados en salas de proyección; si bien esos procesos espectatoriales se han mantenido, quizá con menos fuerza que décadas anteriores, podemos afirmar, junto con otros autores (Casas, 2011; Gubern, 2013; Stam, 2001), que las formas de disfrutar el cine se han ampliado y pluralizado notoriamente.

Para Jenkins (2008) los espectadores formados bajo el paradigma de la cultura digital plantea una visibilidad inédita para el debate académico entorno a las audiencias. De acuerdo con el autor, si los viejos consumidores se suponían pasivos, los jóvenes consumidores de hoy suelen ser activos; si los viejos consumidores eran predecibles y permanecían donde les decías que se quedasen, los jóvenes espectadores ahora son migratorios; si los viejos consumidores eran individuos aislados, en la actualidad los jóvenes consumidores están más conectados socialmente. Si el trabajo de consumidores fue antaño silencioso e invisible, los nuevos y jóvenes consumidores son hoy ruidosos y públicos. Las nuevas herramientas tecnológicas permiten a los jóvenes archivar, comentar, apropiarse y volver a poner en circulación los contenidos cinematográficos (Pujol, 2011). 
Estas condiciones han propiciado que prácticas culturales ligadas al cine como la cinefilia, adquieran nuevos matices. Así dentro de la nueva cultura audiovisual se pone en práctica una nueva cinefilia que lleva la impronta de su tiempo, la sociedad digital, de convergencia mediática y tecnológica (Rodríguez, 2015; Sedeño, 2013), y los jóvenes, como espectadores asiduos, como cazadores furtivos — en palabras de Certeau (1996) - de todo aquello relacionado con sus gustos cinematográficos, son quienes la reactivan.

\section{La cinefilia frente la cultura fan}

Esta popularización digital le dio visibilidad, notoriedad, a un tipo de cinéfilo que anteriormente era excluido de los ámbitos académicos: la figura del fan (Gómez, 2015ª 2015b; Jenkins, 2009; Pujol, 2011), constituyente de una subcultura juvenil que mantiene estrecha relación con las culturas mediáticas, que es consciente de los cambios en los entornos comunicativos porque les da un sentido de participación y de empoderamiento colectivo, que disemina su gusto y se posiciona como grupo por distintas plataformas digitales e interactivas (Gómez, 2015b).

De acuerdo con estudios recientes sobre audiencias juveniles y consumos culturales (Gómez, 2015 a y 2015b; Jenkins, 2008, 2009 y 2010; Pujol, 2011), la cultura fan siempre ha estado relacionada a cierta noción de subcultura juvenil. Si bien el sujeto empieza a vincularse, sentimentalmente primero, a determinados textos mediáticos durante su infancia (programas de televisión, películas, comics) son en los períodos de adolescencia, post-adolescencia, en la transición de la juventud a la adultez donde ese vínculo se fortalece en los grupos de pares que se establecen en los procesos de sociabilidad. Se es fan, se asume como fan de algo cuando se es joven, y si bien ese sentimiento puede permanecer a lo largo del tiempo biográfico, su intensidad puede disminuir (o no) en períodos posteriores relacionados con la madurez biológica (Gómez, 2015ª y 2015b; Pujol, 2011).

Lo anterior nos permite hablar de un tipo particular de audiencia joven en la que el vínculo con los contenidos mediáticos está definido principalmente por un componente sentimental, vivencial y biográfico más que intelectual, a diferencia de la cinefilia tradicional, aunque este último no queda excluido (Jenkins, 2010; Pujol, 2011). Cuando hablamos de cinefilia tradicional nos referimos a aquella que en un segundo momento fue integrada a los ámbitos universitarios, que adoptó los cineclubes y los salas de cine de arte y ensayo como nichos legitimadores, ya señalada en párrafos anteriores.

Para autores como Jenkins (2008) y Scolari (2013) las comunidades de fans -en el que incluiríamos, de acuerdo a lo planteado en el párrafo anterior, a las comunidades cinéfilas - fueron los primeros en apropiarse productivamente de las 
nuevas tecnologías y visibilizarse como colectivos en el ciberespacio. Según los autores una mayoría significativa de comunidades de interés e interpretativas en línea es de esa naturaleza.

Lo anterior nos lleva a otro factor importante, los ámbitos en el que se configuran esos vínculos simbólicos entre el espectador y los textos cinematográficos. A diferencia de la vieja cinefilia, o cinefilia tradicional, el cinéfilo visto desde la perspectiva de la cultura fan trasciende los ambientes académicos o institucionales como nichos fundacionales y reforzadores de ese interés o sentimiento. Si bien, como decíamos, lo generacional es uno de los factores decisivos en la configuración de la cultura fan, no lo es el grado de escolaridad. Ser fan o asumirse como tal es independiente a pertenencias institucionales.

Al respecto, Jenkins $(2010$, p. 31) plantea que las formas de aproximarse a los textos mediáticos, incluido el cine, parte de estas comunidades interpretativas, difieren de las que fomenta el sistema educativo y las que prefiere la cultura institucionalizada y académica no simplemente en las elecciones de sus objetivos (textos propios de la cultura popular o la cultura de masas) o en el grado de su intensidad, sino a menudo en los tipos de habilidades lectoras que emplean, en las maneras en las que los fans cinéfilos abordan los textos. Desde la perspectiva del gusto dominante - apunta Jenkins - los fans parecen ser unos lectores preocupantemente incontrolados, indisciplinados. Los fans abrazan con entusiasmo sus textos preferidos e intentan integrar las representaciones de los medios de comunicación en su propia experiencia social.

La cultura fan implica determinadas formas de consumo. En lo referente al cinéfilo como fan Pujol (2011) nos plantea que la discriminación de unos textos sobre otros es una de las actividades de sus prácticas: hay unos textos o productos cinematográficos dignos de ser objeto de culto y otros que no. La cinefilia fortalecía su gusto más en factores intelectuales que emocionales; el fan, como sugeríamos en el párrafo anterior, configura su vínculo a partir de factores sentimentales más que intelectuales. El cinéfilo fan es visceral, el viejo cinéfilo académico-tradicional es racional. Este componente sentimental establece la diferencia entre las prácticas de consumo propias de la cinefilia tradicional y las de la cinefilia que se construye bajo el paradigma del fan.

Mientras las primeras se realizan en busca de un conocimiento cinematográfico amplio complementario al hecho de ver cine y todo lo que el cine como arte y medio de comunicación implica, las prácticas propias de la cultura fan se focalizan, en aspectos muy específicos del universo cinematográfico; bajo esa lógica el del fan se trata de un consumo muy especializado, concentrado en aspectos muy específicos del cine. El espectador cinéfilo tradicional gusta del cine en general. El espectador joven fan gusta del cine, pero su fijación está dirigida - y extendida a lo largo del 
tiempo biográfico - a textos cinematográficos específicos (o un conjunto de textos) o a signos particulares (o un conjunto de ellos) del universo filmico (un producto en particular, un personaje o un grupo de personajes en especial, un género cinematográfico en específico).

\section{Cinefilia 2.0: una cinefilia que se pluraliza y diversifica}

Esas formas de acercamiento a los contenidos cinematográficos implican ciertas formas de apropiación y productividad. De acuerdo con Pujol (2011) una característica que definen la actividad de los fans es la producción activa de significados que constantemente realizan estos comunidades. Esta continua producción de significados se refiere a la identidad y experiencia social de estos individuos a partir de determinados recursos de la producción cultural. Producción de significados que se hace pública al ser compartida en las redes, generando visibilidad social, de aquí que la discusión y el intercambio de opiniones, apreciaciones y juicios entre los integrantes de estos colectivos sea su característica más distintiva.

Lo anterior trae consigo la instauración de nuevas sensibilidades cinematográficas y permite señalar a Internet como el recurso que catapultó nuevas cinefilias, así en plural (Jullier y Leveratto, 2012). Con ello la cinefilia perdió su aura elitista con la que estuvo ligada durante el siglo pasado — particularmente la posterior al período de la Nueva Ola Francesa-, se vulgarizó, en el sentido estricto del término, diversificándose. El canon francés del gusto cinematográfico (el culto al autor, el rechazo al cine de géneros, los ámbitos académicos como nichos cinéfilos) entró en tensión por el surgimiento y visibilidad de grupalidades que han estado reivindicando su gusto, un gusto - unos gustos - que no busca oponerse sino ampliar aquel espectro cinéfilo con tendencia estéticamente purista y culturalmente excluyente (Pujol, 2011; Jullier y Leveratto, 2012).

El feminismo, la comunidad LBGT+, las minorías étnicas y las tribus mediáticas ya venían causando cierta tensión en ese sentido desde las dos últimas décadas del siglo pasado pronunciando nuevos referentes cinematográficos. Y, claro, nuevos procesos para pronunciarlos: es aquí donde la cultura digital e Internet jugaron un papel decisivo. Lo propio de la cinefilia posmoderna — nos recuerdan Jullier y Leveratto (2012) - es reconocer la importancia de las diferencias de sensibilidades en el interés de la experiencia cinematográfica; así el respeto por el punto de vista del otro en el juicio cinematográfico es aquí, en consecuencia, esencial. El canon cinéfilo heredado de la cultura cinematográfica francesa aludido en apartados anteriores es puesto en cuestión:

La cinefilia moderna, erudita, descansaba en la mirada experta de un lector que era una autoridad en la materia, a menudo con el apoyo del autor, 
[...] Pero la cinefilia posmoderna, marcada por la cultura participativa, la pluralidad de las significaciones y el relativismo, integró la ausencia de una lectura única, y la necesidad de tener en cuenta la palabra de los espectadores, a partir del momento en que participan en la construcción de los acontecimientos. (Jullier y Leveratto, 2012, p. 219)

Los nuevos cinéfilos, se dice, los que se han forjado bajo la cultura digital y las ventajas de la web 2.0, abandonaron las catedrales de la cinefilia privilegiando la comodidad del ordenador y el explorador de mejor rendimiento. Googlean títulos, descargan películas, las archivan y las comparten, las remezclan si así lo desean, se apropian de ellas, socializan su gusto por ellas en las redes sociales, en blogs o videoblogs, organizan y participan en conversaciones y debates en las comunidades virtuales. El tamaño de la pantalla, por cierto, les es indiferente siempre y cuando pueda perpetuarse el placer de estar atentos a la narración audiovisual y compartir la experiencia (Pujol, 2011; Jullier y Leveratto, 2012).

\section{Conclusiones}

Como hemos podido ver la cinefilia hoy ha adquirido un sentido algo diferente. Ha experimentado algunos desplazamientos como el cine mismo: las conversaciones sobre cine han dejado atrás los espacios tradicionales (las universidades, las revistas especializadas en cine) para diseminarse a través de redes y plataformas digitales. La pronunciación del juicio estético se ha democratizado y ello le ha impreso a la cinefilia entendida como fenómeno un carácter más cercano a lo popular.

¿Estamos ante una cinefilia devaluada? Con el presente trabajo hemos intentado demostrar lo contrario. Se trata, en todo caso, de maneras de satisfacer el gusto y cultivar el placer por el cine muy diferentes a las de la segunda mitad del siglo xx, y más acordes a la sociedad digital. Es en definitiva una forma diferente de alimentar ese conocimiento por parte de aquellos jóvenes que nacieron en esa transición entre un viejo y nuevo siglo.

Como todos lo fenómenos que transmutan de acuerdo a cada contexto y avances tecnológicos, la nueva cinefilia que hemos denominado 2.0 y las demás formas de consumo cultural demandan cambios, también, en las perspectivas al momento de abordar su estudio. Por ello es importante tener en cuenta que:

Estudiar los modos de afición, consumo y creación de opinión en torno al cine en el mundo actual supone superar los prejuicios impuestos por esa cinefilia surgida en los años cincuenta. Es preciso estudiar el modelo de espectador/espectadora que ha surgido en los últimos años, ver cómo este mo- 
delo ha conquistado determinados ángulos de la crítica y ha dado paso a otras opiniones, otros gustos y, sobre todo, otras formas de ver. (Pujol, 2011, p. 15)

Hay quienes piensan que el término cinefilia 2.0 solo es una etiqueta de marketing digital. Puede ser, pero en estas reflexiones no hemos encontrado un mejor término para designar ese cambio de paradigma.

\section{Referencias bibliográficas}

Ardévol, E. et al. (2010). Prácticas creativas y participación en los nuevos medias. Cuaderns del CAC, número 34, vol. 13. 27-37.

Azatto, M. (2011). Funciones de la imagen digital en la educación: una propuesta metodológica para la escritura y lectura de la imagen en pantallas instruccionales (Tesis doctoral). Universidad de Barcelona, Barcelona, España.

Brea, J. (2007). Cultura_RAM. Barcelona: Gedisa.

Bourriaud, N. (2009). Postproducción. La cultura como escenario. Buenos Aires: AH.

Casas, Q. (2011). Análisis y crítica audiovisual. Barcelona: UOC.

Certeau, M. (1996). La invención de lo cotidiano. 1. Las artes de hacer. México: Universidad Iberoamericana.

Gómez, H. (2015a), Estar en otra parte: tiempos de crecer siendo un fan. Cuatro textos sobre traslados en la cultura y la comunicación. León, Gto: Universidad Iberoamericana León.

Gómez, H. (2015b). Hacer vida en las pantallas: de fans en tiempos del cine después del cine. Post-cines, post-subculturas, post-fandoms. Versión. Estudios de comunicación y política. Num. 36. 42-53.

Gubern, R. (2000). El eros electrónico. Madrid: Ed. Taurus.

Gubern, R. (2013). Cultura audiovisual. Madrid: Cátedra.

Jenkins, H. (2008). Convergence culture. La cultura de la convergencia de los medios de comunicación. Barcelona: Paidós.

Jenkins, H. (2009). Fans, blogueros y videojuegos. La cultura de la colaboración. Barcelona: Paidós.

Jenkins, H. (2010). Piratas de textos. Fans, cultura participativa y televisión. Barcelona: Paidós.

Jullier, L. y Leveratto, J. (2012). Cinéfilos y cinefilias. Buenos Aires: La marca editora.

Lipovetsky, G. y Serroy, J. (2009). La pantalla global. Cultura mediática y cine en la era hipermoderna. Barcelona: Anagrama.

Octobre, S. (2019). ¿Quién teme a las culturas juveniles? Las culturas juveniles en la era digital. Barcelona: Océano.

Pujol, C. (2011). Fans, cinéfilos y cinéfagos. Una aproximación a las culturas y gustos cinematográficos. Barcelona: UOC. 
Rodríguez, Aarón (2015). La cinefilia 2.0 y el frameo: Apuntes teóricos sobre el collage visual en la nueva crítica cinematográfica. Revista Estrategias, Tendencias e Innovación en Comunicación. No- 10. 99-113.

Scolari, C. (2008). Hipermediaciones. Elementos para una teoría de la comunicación digital interactiva. Barcelona: Gedisa.

Scolari, C. (2013). Narrativas transmedia. Cuando todos los medios cuentan. Barcelona: Deusto.

Sedeño, A. (2013). Nueva cinefilia: reflexiones sobre la transformación de las prácticas cinéfilas por las nuevas tecnologías de la contemporaneidad. Razón y Palabra, n. ${ }^{\circ} 83$. 1-13.

Stam, R. (2001). Teorías del cine. Una introducción. Barcelona: Paidós. 